\title{
Propuesta de Índice de acceso y tenencia de las TIC en centros educativos públicos en Costa Rica
}

\section{Proposal for an index of access and ownership of ICT in Public Educational Centers in Costa Rica}

\section{Proposta de um índice de acesso e propriedade das TICs em centros educacionais públicos na Costa Rica}

\author{
Melissa Edith Valverde-Hernández \\ Universidad de Costa Rica \\ San José, Costa Rica \\ melissa.valverde_h@ucr.ac.cr \\ (D) ORCID: https://orcid.org/0000-0003-0669-6320 \\ Cristina Paniagua-Esquivel \\ Universidad de Costa Rica \\ San José, Costa Rica \\ cristina.paniagua@ucr.ac.cr \\ (D) ORCID: https://orcid.org/0000-0003-4046-6799
}

Recibido - Received - Recebido: 25/ 07 / 2021 Corregido - Revised - Revisado: 9 / 10 / 2021 Aceptado - Accepted - Aprovado: 12 / 10 / 2021

\begin{abstract}
Resumen: El presente artículo presenta una propuesta de índice de acceso y tenencia de tecnologías en centros educativos públicos, para cuantificar la brecha existente entre estos. Se proponen dos indicadores: acceso y tenencia de tecnología digital para construir el Índice de Acceso y Tenencia TIC (IAT TIC). Posterior al diseño del índice, se aplicará a los datos del I Censo Nacional de Tecnologías Digitales en Educación, realizado por el Ministerio de Educación Pública de Costa Rica con la colaboración de la Universidad de Costa Rica. En este censo se registró el dato de 3.733 centros educativos y entre los resultados se destacan magnitudes promedio menores en direcciones regionales como Sulá $(2,5)$ y Coto $(4,19)$ y mayores en regiones centrales del país. Por lo tanto, se concluye que el IAT-TIC es un índice consistente, el cual cuantifica una brecha en el acceso y tenencia de TIC en los centros educativos públicos costarricenses.
\end{abstract}

Palabras clave: Brecha digital, indicadores educativos, tecnología de la información, acceso, tecnología, educación pública.

Summary: This article presents a proposal for an index of access and ownership of technologies in educational centers, to quantify the existing gap between them. Two indicators are proposed: access and possession of digital technology to build the ICT Access and Tenure Index (IAT TIC). After the design of the index, it will be applied to the data of the I National Census of Digital Technologies in Education, carried out by the Ministry of Public Education of Costa Rica in collaboration with the University of Costa Rica. This census includes the data of 3733 educational centers. The results include lower average magnitudes such as Sulá (2.5) and Coto (4.19) and higher ones in central regions of the country. Therefore, it is concluded that the IAT-ICT is a consistent index, which quantifies a gap in access and possession of ICT in Costa Rican public educational centers.

Key Words: Digital divide, Educational indicators, Information technology, Access, Technology Public education

Resumo: Este artigo apresenta uma proposta para um índice de acesso e posse de tecnologias em centros educacionais, a fim de quantificar a lacuna existente entre eles. Dois indicadores são propostos: acesso e propriedade da tecnologia digital para construir o Índice de Acesso e Posse, TIC por suas siglas em inglês,((IAT ICT). Após o desenho do índice, ele será aplicado aos dados do $1^{\circ}$ Censo Nacional de Tecnologias Digitais na Educação, realizado pelo Ministério da Educação Pública da Costa Rica, em colaboração com a Universidade da Costa Rica. Neste censo, foram registrados dados de 3.733 centros educacionais. Entre os resultados, as 
magnitudes médias foram menores nas Diretorias Regionais de Educação, como Sulá $(2,5)$ e Coto (4,19), e maiores nas regiões centrais do país. Portanto, conclui-se que o IAT-ICT é um índice consistente, que quantifica uma lacuna no acesso e posse das TIC nas escolas públicas costarriquenhas.

Palavras-chave: Gap digital, Indicadores educacionais, Tecnologia da informação, Acesso, Tecnologia, Tecnologia Educação pública.

\section{INTRODUCCIÓN}

Desde los años 80 (Lugo, 2016), el ingreso de las tecnologías de la información y comunicación (TIC) y tecnologías digitales (TD) en la cotidianidad, han realizado cambios en la forma en cómo se relacionan y comunican las personas y, pertinente a este artículo, enseñan y aprenden (Cuevas, Álvarez y Raviaso, 2009). Esta inserción ha traído consigo una serie de beneficios, pero también ha generado una brecha tanto entre países, como entre centros educativos dentro de cada uno.

La brecha digital se puede presentar en varias dimensiones, según Cabero-Almenara y Ruiz-Palmero (2018) señalan que incluye el no tener acceso a la tecnología, pero también hay una que se da cuando se tiene acceso, pero no conocimiento para usarlas. A esto se les conoce como brecha de primer nivel y segundo nivel, respectivamente (Moreira, Palomares, Serrano y López, 2017). Cabero-Almenara y RuizPalmero (2018) concluyen que esta última brecha es en la que se deberían enfocar los estudios; sin embargo, no en todos los países (incluido Costa Rica) existe un índice que permita cuantificar la diferencia entre la cobertura de los elementos más básicos de la brecha. Lo anterior resulta necesario, para enfocar los esfuerzos en aquellos centros que requieran atención más urgente.

En la búsqueda de estudios previos sobre brecha, se halló que la mayoría están enfocados en hogares; sin embargo, se toman en cuenta, porque plantean las bases para comprender mejor sus implicaciones. Primeramente, se encuentra el Ministerio de Ciencia y Tecnología de Costa Rica (2019), el cual muestra indicadores de acceso (telefonía móvil, acceso a computadora, a internet y la banda ancha (Mbps por persona)). Otro estudio es el de Chanto y Loáiciga (2020), quienes evaluaron la brecha digital en personas universitarias en Liberia. En recursos, tomaron en cuenta tenencia de computadora (portátil y de escritorio), conexión a internet y tabletas.

Moreira et al. (2017) identificaron criterios para caracterizar y medir la brecha digital y, posteriormente, mostraron el nivel en Ecuador. Se plantean tres etapas para valorar la inserción de las TIC y, en la primera etapa, se destaca el nivel de infraestructura y acceso. Por otro lado, González-Zabala y Sánchez-Torres (2012) efectuaron un análisis de variables e indicadores para caracterizar las sociedades de la información. Entre las dimensiones están la infraestructura, la cual se toma en cuenta como parámetro para medir la brecha digital. También, Hawash y Lang (2020) estudiaron varios países y concluyen que es importante considerar el capital físico como infraestructura, líneas telefónicas o electricidad para aprovechar los beneficios de las TIC.

Internacionalmente, Morales (2020) analiza el estado de las brechas digitales en México, para lo cual tomó en cuenta indicadores como el uso de tecnologías básicas (computadoras, televisión y conexión a internet). Gómez, Alvarado, Martínez y Díaz de León (2018) estudiaron también la brecha digital en México y propusieron que, a nivel de acceso, se debe tomar en cuenta el motivacional (imaginarios sobre la posibilidad de uso/no uso), físico (infraestructura tecnológica y posibilidad de adquisición) y alfabetización digital. Con esto, plantearon un índice de desarrollo de TIC que incluía un uso bajo, mediobajo, medio-alto y alto, según el puntaje de las personas.

Los índices son medidas compuestas que relacionan variables o constructos de distinta naturaleza (Gómez, 1997, como se citó en Montero, 2018). Por otro lado, un indicador es la forma en que se operacionaliza una variable, la cual permite efectuar un cálculo empírico (Montero, 2018). Tiana (2021) define 
que un indicador es un procedimiento o instrumento que permite representar o analizar información relevante, la cual muchas veces es abstracta. Los resultados de la aplicación de los indicadores pueden sustentar o guiar políticas educativas. Núñez (2018) señaló que no había un sistema de indicadores para medir beneficios reales que dan las TD a la educación y la actualidad, esto se mantiene. Es importante que se desarrolle uno adaptado a Costa Rica; pues la elaboración y el cálculo de indicadores e índices contribuyen a la información objetiva y pertinente para las personas e instituciones tomadoras de decisión. El índice parte de lo propuesto por la Organización de las Naciones Unidas para la Educación, la Ciencia y la Cultura (UNESCO, 2009), que señala los requerimientos más básicos para la integración de TIC educación (ver figura 1).

Figura 1. Evolución en el tiempo de las necesidades de información de los sistemas educativos según el nivel de penetración de las TIC.

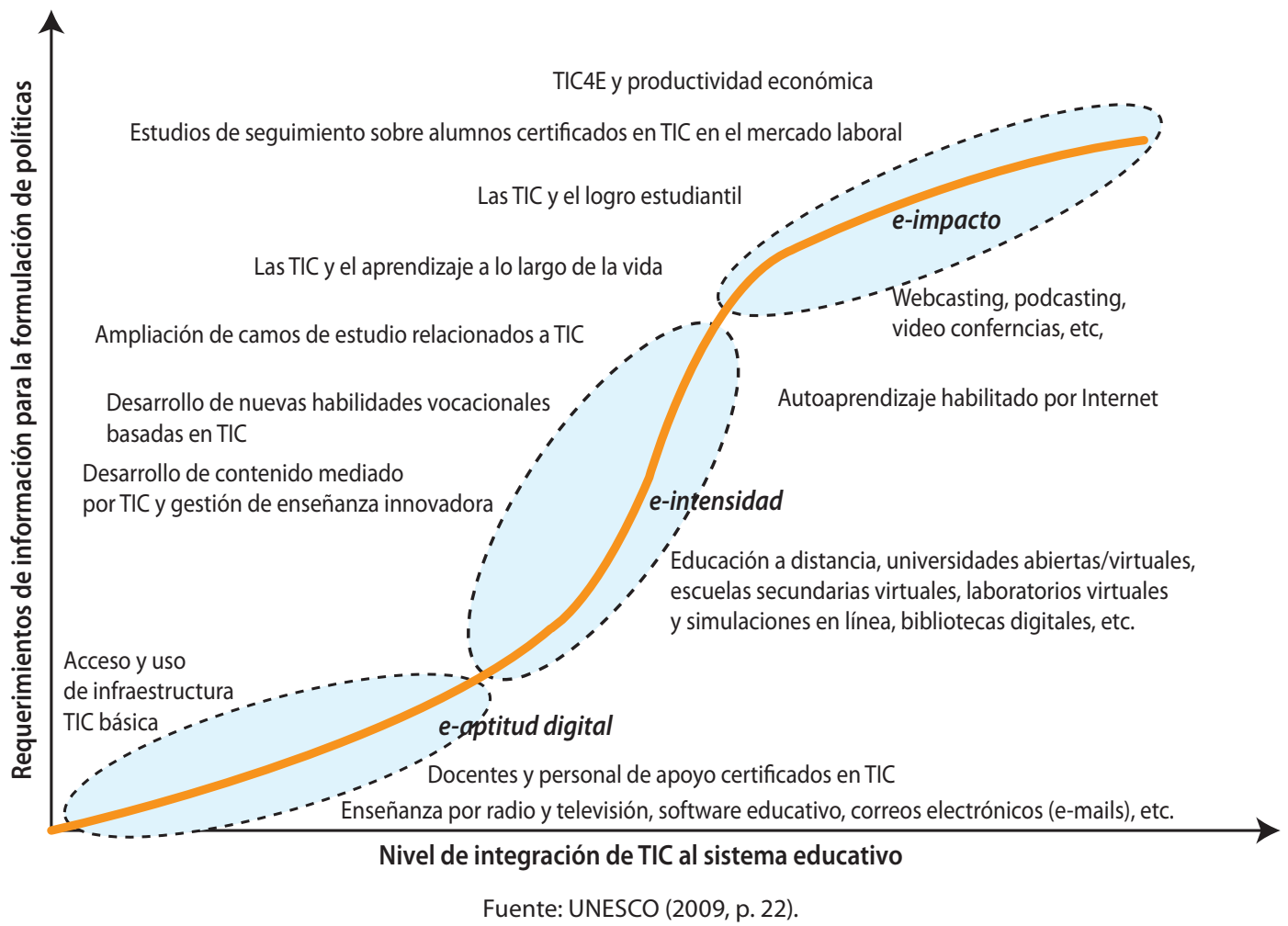

En Costa Rica existen iniciativas que buscan ubicar la educación en el nivel "e-intensidad". Por ejemplo, en el 2019 se instalaron más de 36 mil equipos (Carreño, 2020); sin embargo, aún se encuentran retos como el acceso a la conectividad, los cuales generan una brecha enorme (Ramírez, 2021) que convierten a Costa Rica en un país emergente. Estos países se caracterizan por tener conciencia de los beneficios, pero con limitaciones en factores clave, como conectividad y financiamiento (Lugo y Kelly, 2010). La medida que se propone resumirá información vinculada al acceso a conexión eléctrica e internet, participación en programas de innovación educativa y nivel de acceso a los dispositivos, para conocer los centros sobre los cuáles se debe incidir. Para esto, se proponen dos dimensiones: acceso y tenencia, con sus respectivos indicadores (ver Tabla 1). 
TABLA 1

Dimensiones e indicadores del Índice de acceso y tenencia de TIC de centros educativos públicos del MEP, 2021

\begin{tabular}{|c|c|}
\hline Dimensión & Descripción conceptual \\
\hline \multirow{3}{*}{ Acceso } & $\begin{array}{l}\text { Infraestructura. Se centra en servicios básicos para poder acceder a la tecnología, como electricidad y con- } \\
\text { diciones del edificio. También si cuenta con servicio de biblioteca o laboratorio de cómputo. }\end{array}$ \\
\hline & Cobertura de internet. Incluye no solo si tiene o no conexión, sino la velocidad y la percepción de la calidad. \\
\hline & $\begin{array}{l}\text { Participación en programas de TD. El tener la infraestructura básica y la cobertura a internet, les permite } \\
\text { acceder a programas de acceso a equipos. }\end{array}$ \\
\hline \multirow{2}{*}{ Tenencia } & $\begin{array}{l}\text { Dispositivos básicos. Dispositivos que también se requieren para mediación pedagógica, pero no esencia- } \\
\text { les, como: televisión, video beam, impresora, fax, escáner, fotocopiadora y cámara. }\end{array}$ \\
\hline & $\begin{array}{l}\text { Dispositivos avanzados. Son los dispositivos fundamentales para el proceso de enseñanza-aprendizaje. Se } \\
\text { incluye la tenencia de computadoras (escritorio o portátil) y tabletas. }\end{array}$ \\
\hline
\end{tabular}

Fuente: elaboración propia.

El acceso a la infraestructura es un indicador primario (UNESCO, 2009); sin una infraestructura adecuada, sin ciertas condiciones mínimas, no es posible la innovación. Entre los requerimientos mínimos se hallan la conexión eléctrica, tener redes de comunicación, espacios físicos como bibliotecas y mobiliario (Severin, 2011). Sin embargo, no solo hay que tomar en cuenta la cantidad, sino la calidad de las instalaciones y recursos disponibles (Vázquez y Ramírez, 2016). Hawash y Lang (2020) indican que la falta de infraestructura o fuentes de energía eléctrica estables disminuye las oportunidades de tener mejores y más rápidos sistemas de información y comunicación.

Severin (2011) plantea que dentro de esta infraestructura existen espacios físicos como bibliotecas. En el 2010 estas autoras señalaron el laboratorio de cómputo como el lugar usual para disponer de la tecnología. En Costa Rica existen las BiblioCRA, las cuales son bibliotecas que se han convertido en Centros de Recursos para Aprendizaje e incorporan variedad de estos recursos, como computadoras, cámaras, tablets, proyectores y conexión a internet (Brenes, 2020).

En relación con el internet, Lugo y Kelly (2011) plantean que en un nivel inicial la conexión se efectúa con una línea telefónica; mientras, en un nivel avanzado, hay varios tipos de conexión que permiten mayor velocidad y calidad (ej.: banda ancha y conexiones inalámbricas). Acceder a la red es un reto, y hay que tomar en cuenta no solo el tener, sino si la conexión es estable (Severin, 2011).

Con respecto a la velocidad, la Comisión Económica para América Latina y el Caribe (CEPAL, 2020) señala que la velocidad de banda ancha se puede clasificar en Baja (5,5 Mbps), Media (18,5 Mbps) y Alta (más de $25 \mathrm{Mbps}$ ). Estos datos dependen de las funcionalidades que permite y se plantearon al tomar en cuenta las necesidades por causa de la pandemia que produce la COVID-19, como alternativa en la educación a distancia.

En la participación en programas hay que valorar la asociación entre las instituciones públicas y privadas, así como la inversión que estas pueden dar en la implementación de tecnología como indicador del nivel de incorporación de las TIC (Vázquez y Ramírez, 2016). En el caso de Costa Rica, existen varios proyectos de innovación educativa (ver Cuevas y Núñez. 2016 y Solórzano, 2008, para el resumen de estos). Estos proyectos se centran también en la capacitación docente.

Con respecto a la tenencia, dentro de los parámetros que se consideran importantes en recursos TIC, es la variedad de dispositivos. A pesar de que la computadora tiene un lugar importante, hay otros dispositivos tecnológicos que aportan a la educación. UNESCO (2009) enlista una serie de dispositivos que deberían tener los centros educativos; entre estos se hallan: la radio, las pizarras electrónicas y los televisores. En el caso de estos últimos, es uno de los medios de comunicación más utilizados en la cotidianidad; por lo tanto, su uso en el sistema educativo puede ser una herramienta para unir el centro 
con la sociedad (López-Mondéjar, 2018). También se utilizan las cámaras digitales, pizarras, móviles y escáneres (Lugo y Kelly, 2011). En el nivel inicial de inserción de tecnología, hay computadoras para el equipo directivo y computadoras en el laboratorio de informática (Lugo y Kelly, 2011), en el intermedio hay computadoras en salones de clase, y estas (junto con impresoras) son accesibles para docentes y estudiantes y, en avanzado, hay un dispositivo de uso personal para docentes y estudiantes.

Por otro lado, las tabletas han tenido un crecimiento como herramienta didáctica y son parte del aprendizaje móvil, lo cual permite la disminución de brecha; pues el estudiantado puede acceder a las TIC, sin incurrir en el costo de una computadora (Fernández, 2016). Esta misma autora señala una serie de funcionalidades didácticas, que van desde herramientas de ofimática hasta producción y edición de contenidos (ej. videos).

Este artículo tiene como objetivos construir un índice de brecha tecnológica en el acceso y uso de tecnología, y aplicarlo a los resultados del I Censo Nacional de TD en Educación, realizado por el Ministerio de Educación Pública de Costa Rica (MEP). Específicamente, se analizarán los datos de los cuestionarios sobre infraestructura. Este cuestionario incluía preguntas sobre condiciones generales, instalación, acceso, tenencia y participación de proyectos. Con esto se responderá a la pregunta: ¿cuál es el nivel de brecha en uso y acceso tecnológico en centros educativos públicos de Costa Rica?

\section{MATERIALES Y MÉTODOS}

El estudio es de enfoque cuantitativo, pues se centra en la generalización de los resultados métricos y la construcción de una línea base a nivel país. Para esto se realizó un Censo a nivel nacional, en el cual participaron las personas directoras de 3733 centros educativos (cobertura 80\% de 4780 centros), de las 27 Direcciones Regionales de Educación (DRE) del país. Las DRE son una división administrativa y de atención a comunidades educativas, las cuales agrupan a todos los centros educativos del país (MEP, s.f.). La población de estudio son todos los centros educativos públicos del país de todos los niveles educativos, en donde la persona directora es informante sobre las condiciones de infraestructura tecnológica del centro.

Para la construcción del instrumento, se consultó a personas expertas en la temática y pruebas piloto con personas directoras, para evaluar el contenido y la redacción de las preguntas. El cuestionario se realizó a finales de 2016por medio de la plataforma de encuestas en línea LimeSurvey. La persona directora de cada centro educativo realizaba el registro a participar y por correo electrónico (la participación se realizó mediante una directriz institucional), se efectuaba una verificación del registro con la nómina del personal del MEP y, seguidamente, se enviaba una notificación para el completado del "Cuestionario de capacidades de Infraestructura física, conectividad, equipo y mobiliario de los Centros Educativos". El cuestionario se encuentra conformado por los siguientes módulos:

1. Generalidades del centro educativo.

2. Proyectos activos en el centro educativo.

3. Infraestructura general del centro educativo.

Esta consulta tenía el propósito de construir una línea base nacional que dé cuentas del estado de situación de las TD en los niveles educativos: Preescolar, I, II y III Ciclo y Educación Diversificada. Además, de servir de insumo para orientar investigaciones educativas y apoyar en la toma de decisiones en todos los niveles del sistema educativo.

Por otro lado, para la construcción de los índices, se siguieron algunas fases que plantea Babbie (2000), entre ellas: 
1. Base teórica: se realizó una búsqueda de teorías y antecedentes sobre índices relacionados. Esto llevó a la construcción de un marco teórico conceptual, el cual permitió la descripción de las posibles variables e indicadores.

2. Operacionalización de las variables: a partir de la base teórica, se seleccionaron aquellas variables pertinentes y se plantearon de manera operativa.

3. Relaciones bi-variadas: se examinan las relaciones entre las variables e indicadores.

4. Puntuación de los indicadores: en esta fase se asignan puntajes a los indicadores.

5. Fórmula de cálculo del índice: se define una fórmula para cuantificar el índice.

6. Validación interna-externa: se realizan técnicas estadísticas y se contrasta con información externa para la validación del índice.

En relación con el procedimiento de análisis previamente se elaboraron tablas y gráficos a nivel descriptivo y también se implementaron pruebas y técnicas estadísticas para la validación interna del índice. Se utilizó el software estadístico SPSS y lenguaje estadístico R. Asimismo, se establece un nivel de significancia del $5 \%$.

\section{DISCUSIÓN DE RESULTADOS}

El presente artículo busca construir un índice de brecha tecnológica en el acceso y uso de tecnología, para aplicarlo a los resultados de infraestructura del I Censo Nacional de TD en Educación. Con respecto a las condiciones generales del edificio (ver Figura 2), se encuentra que un porcentaje alto de centros (40\%) reporta que requiere mejoras o está en condiciones aceptables (36\%). Por otro lado, el $96 \%$ de los centros educativos tienen instalación eléctrica.

Figura 2. Costa Rica: porcentaje de centros educativos según las condiciones reportadas de los edificios por la persona directora, MEP, 2016

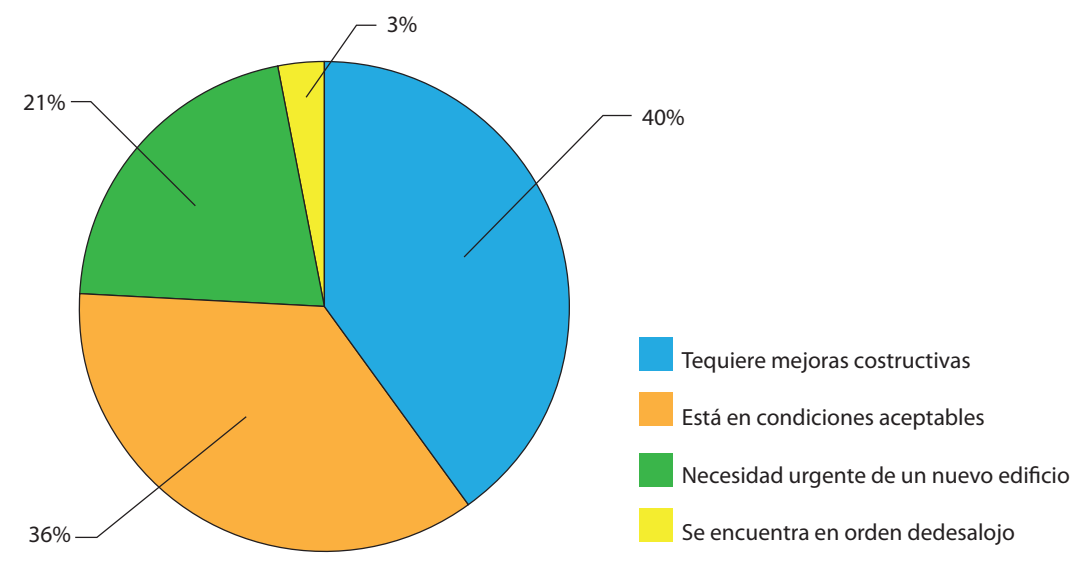

Fuente: elaboración propia.

Se consultó por los diferentes servicios del centro y de la totalidad, el 31\% reporta tener laboratorio de cómputo, $20 \%$ biblioteca, $9 \%$ algún otro tipo de laboratorio y $8 \%$ tiene BiblioCRA. Asimismo, el 75,61\% responde que tienen conexión a internet. Al consultarles por la velocidad, 33,08\% tienen entre $2 \mathrm{Mbps}$ y 4 Mbps; seguido están las conexiones de 1 Mbps (14,46\%), 10 Mbps (13,52\%) y 8 Mbps (8,05\%). Los 
centros que reportan tener entre $512 \mathrm{Kbps}, 6 \mathrm{Mbps}$ y $128 \mathrm{Kbps}$, son muy similares (6,91\%, 6,74\% y 6,04\%). Con respecto a los dispositivos, el que más centros señala tener es la impresora (94,3\%), seguido de al menos un proyector $(77,45 \%)$, al menos un escáner $(73,01 \%)$ y televisor $(49,72 \%)$.

Finalmente, con equipos en centros educativos que sean de su propiedad, la mayoría reporta que no tiene computadoras de escritorio para estudiantes (64,78\%), de escritorio para estudiantes en el aula (90,86\%), portátiles para estudiantes $(56,33 \%)$, computadora personal para cada docente $(65,67 \%)$ y tabletas (87,72\%). Hay que considerar que la pregunta indica "que son propiedad del centro"; por lo tanto, si el centro no considera propio el dispositivo otorgado por algún programa, marcará "No hay". La única excepción son las computadoras portátiles. En esta pregunta no se especifica que sean del centro; por ello se espera que se incluyan las que no son propiedad.

A continuación, se presentan los resultados del desarrollo del índice. La Figura 3 resume esquemáticamente los conceptos y las variables para cuantificar el acceso y tenencia de TIC en los centros educativos.

Figura 3. Esquema de los componentes que se asocian a la conceptualización de acceso y tenencia TIC en centros educativos

Acceso y tenecia de TIC

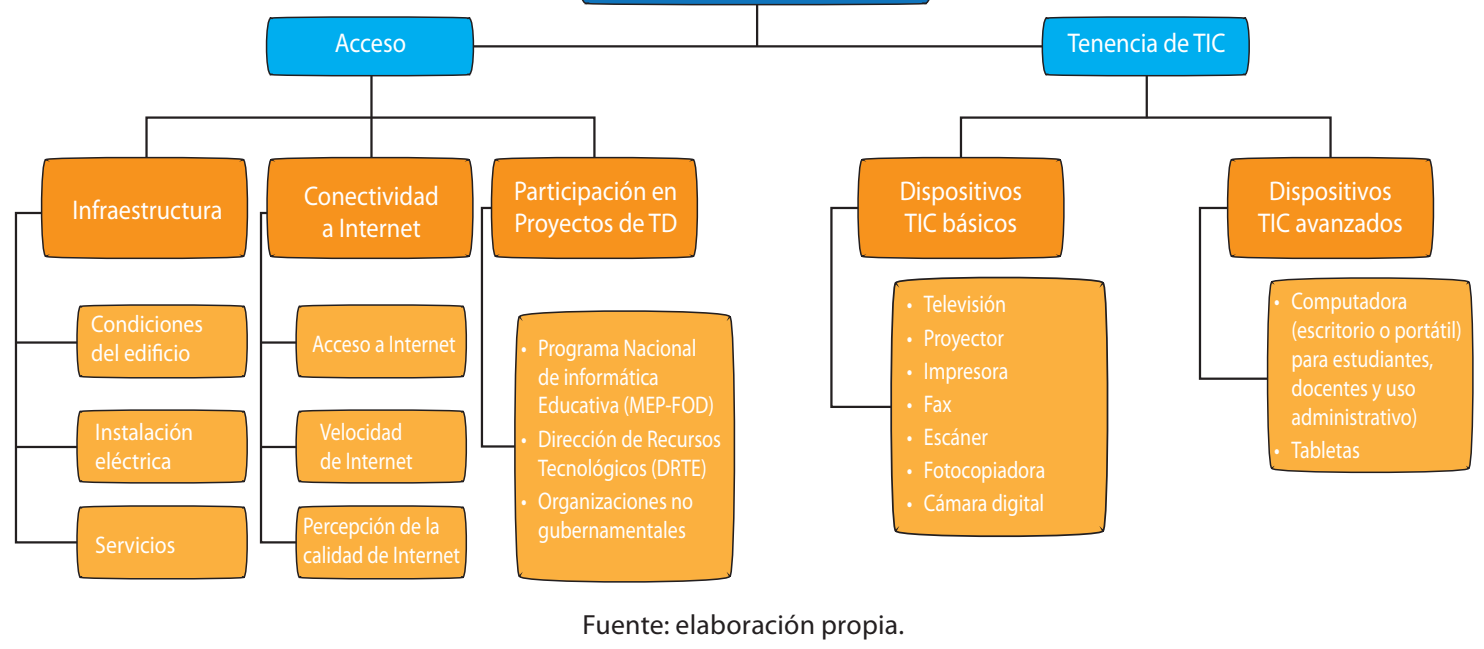

El indicador de acceso contempla componentes generales asociados a la infraestructura, la conectividad de internet y la participación en al menos un proyecto de TD del centro educativo. En la Tabla 2, se describe la operatividad de las variables del indicador. En la parte de infraestructura se toma en cuenta las condiciones generales que tiene el edificio del centro educativo; además, se valora la tenencia y la calidad de la instalación eléctrica del centro y se considera si posee biblioteca y un laboratorio de cómputo. En conectividad, se considera el acceso a internet, la velocidad y la percepción de calidad de la conexión; asimismo, si el centro educativo participa en al menos un proyecto de TD. 
TABLA 2

Costa Rica: Componentes y puntuación de las variables del indicador de Acceso en centros educativos públicos, MEP

\begin{tabular}{|c|c|c|c|c|}
\hline \multirow{2}{*}{ Componente } & \multirow{2}{*}{ Variable } & \multirow{2}{*}{ Valoraciones } & \multicolumn{2}{|c|}{ Puntuación } \\
\hline & & & Min. & Máx. \\
\hline \multirow{6}{*}{ Infraestructura } & \multirow{3}{*}{ Condiciones del edificio } & 0-Necesidad urgente de edificio nuevo/ Orden de desalojo & \multirow{3}{*}{0} & \multirow{3}{*}{2} \\
\hline & & 1-Requiere mejoras constructivas & & \\
\hline & & 2-Está en condiciones aceptables & & \\
\hline & \multirow{2}{*}{ Instalación eléctrica } & $\begin{array}{l}\text { 0-No tiene instalación } \\
\text { 1-Tenencia de instalación }\end{array}$ & 0 & 1 \\
\hline & & $\begin{array}{l}\text { Calidad de la instalación (0- No tiene, 1-Pésima, 2-Muy mala, 3-Mala, } \\
\text { 4-Regular, 5-Buena, 6-Muy buena, 7-Excelente) }\end{array}$ & 0 & 7 \\
\hline & Servicios & $\begin{array}{l}\text { 0-No tiene biblioteca y laboratorio de cómputo } \\
\text { 1-Tiene solo biblioteca o laboratorio de cómputo } \\
\text { 2-Tiene ambos servicios }\end{array}$ & 0 & 2 \\
\hline \multirow{6}{*}{ Conectividad } & Conexión a Internet & $\begin{array}{l}\text { 0-No tiene acceso } a \text { internet } \\
\text { 1-Acceso } a \text { internet }\end{array}$ & 0 & 1 \\
\hline & \multirow{5}{*}{ Velocidad de la conexión } & 0-No tienen & \multirow{4}{*}{0} & \multirow{4}{*}{3} \\
\hline & & 1-Menos de 1 MB & & \\
\hline & & 2-De $1 \mathrm{MB}$ a $6 \mathrm{MB}$ & & \\
\hline & & 3-Más de 6 MB & & \\
\hline & & $\begin{array}{l}\text { Calidad de conexión (0- No tiene, 1-Pésima, 2-Muy mala, 3-Mala, 4- } \\
\text { Regular, 5- Buena, 6-Muy buena, 7-Excelente) }\end{array}$ & 0 & 7 \\
\hline Participación & Proyectos de TD & $\begin{array}{l}\text { 0-No participa en proyectos } \\
\text { 1-Al menos participa en un proyecto de TD }\end{array}$ & 0 & 1 \\
\hline
\end{tabular}

Fuente: elaboración propia.

Este indicador tiene un puntaje máximo de 25 puntos; sin embargo, se realiza un escalamiento del puntaje obtenido a un rango de 0 a 1. Posteriormente, se considera una ponderación de $60 \%$ de indicador de acceso del índice total, pues si un centro educativo no cuenta con las condiciones mínimas de infraestructura, conectividad y proyectos de TD, difícilmente el centro educativo tendrá a su disposición dispositivos TIC.

El índice contempla otro indicador vinculado con la tenencia de dispositivos TIC. Anteriormente, se mencionó que la tenencia de estos dispositivos contribuye a la mediación pedagógica y al acceso a la sociedad de la información. Este tiene dos componentes en los cuales se considera la tenencia de dispositivos básicos y dispositivos avanzados. La Tabla 3 muestra los componentes y las puntuaciones del indicador. 
TABLA 3

Costa Rica: Componentes y puntuación de las variables del indicador de Tenencia TIC en centros educativos públicos, MEP

\begin{tabular}{|c|c|c|c|c|c|}
\hline \multirow{2}{*}{ Componente } & \multirow{2}{*}{ Variable } & \multirow{2}{*}{\multicolumn{2}{|c|}{ Valoraciones }} & \multicolumn{2}{|c|}{ Puntuación } \\
\hline & & & & Min. & Máx. \\
\hline \multirow{7}{*}{$\begin{array}{l}\text { Dispositivos } \\
\text { básicos }\end{array}$} & \multirow{7}{*}{$\begin{array}{c}\text { Tenencia de dispositivos } \\
\text { básicos TIC }\end{array}$} & Televisión & \multirow{7}{*}{$\begin{array}{l}\text { 0- No tiene, 1-Tiene } \\
\text { Se realiza un conteo } \\
\text { de la tenencia total de } \\
\text { dispositivos }\end{array}$} & \multirow{7}{*}{0} & \multirow{7}{*}{7} \\
\hline & & Proyector multimedia & & & \\
\hline & & Impresora & & & \\
\hline & & Fax & & & \\
\hline & & Escáner & & & \\
\hline & & Fotocopiadora & & & \\
\hline & & Cámara digital & & & \\
\hline \multirow{5}{*}{$\begin{array}{l}\text { Dispositivos } \\
\text { avanzados }\end{array}$} & \multirow{5}{*}{$\begin{array}{c}\text { Tenencia de dispositivos } \\
\text { avanzados TIC }\end{array}$} & \multicolumn{2}{|c|}{ Computadora (escritorio o portátil) } & 0 & 8 \\
\hline & & - para estudiantes & $\begin{array}{l}\text { 0-No hay, 1- Hay, pero sin } \\
\text { internet/no indica, 2- Hay } \\
\text { con conexión a internet }\end{array}$ & & \\
\hline & & - para docentes & $\begin{array}{l}\text { 0-No hay, 1- Hay, pero sin } \\
\text { internet/no indica, } 2 \text { - Hay } \\
\text { con conexión a internet }\end{array}$ & & \\
\hline & & - para uso administrativo & $\begin{array}{l}\text { 0-No hay, 1- Hay, pero } \\
\text { sin internet/no indica, } \\
2 \text { - Hay con conexión a } \\
\text { internet }\end{array}$ & & \\
\hline & & Tabletas disponibles & $\begin{array}{l}\text { 0-No hay, 1- Hay, pero } \\
\text { sin internet, 2- Hay con } \\
\text { conexión a internet }\end{array}$ & & \\
\hline
\end{tabular}

Fuente: elaboración propia.

El indicador de Tenencia TIC se conforma de quince puntos, de igual forma se procede a realizar un escalamiento de la puntuación a un rango de 0 a 1. Este contempla el $40 \%$ restante del total del índice.

El índice de acceso y tenencia TIC (IAT TIC) es una medida que cuantifica las condiciones de acceso del centro educativo y la tenencia de dispositivos TIC, con el fin de conocer los requerimientos de información de TD para formulación de políticas y programas, así como también identificar el avance del nivel de integración de TIC en el sistema educativo costarricense. Este índice se halla conformado de los dos indicadores descritos previamente, donde la mayor ponderación se asocia a las condiciones de acceso del centro educativo para la incorporación y tenencia de TIC y la ponderación restante a la tenencia de dispositivos TIC básicos y avanzados. Con los dos indicadores definidos, la fórmula de cálculo del índice se define de la siguiente manera:

\section{IAT TIC $=6^{*}$ Indicador de Acceso $+4 *$ Indicador de Tenencia TIC}

Este índice se encuentra en un rango de 0 a 10, donde 0 es la puntuación más baja y 10 es la máxima. Se realiza un análisis descriptivo de los indicadores y el índice; en la Tabla 4 se muestra que ambos indicadores contemplan los valores mínimos y máximos del rango establecido, lo cual evidencia que existen centros educativos con la puntuación más alta (6 puntos) en las condiciones de acceso y también en la tenencia de dispositivos (4 puntos). En el indicador de acceso se tiene una puntuación promedio de 3,27, lo cual indica que los centros educativos públicos presentan condiciones moderadas en relación con la infraestructura, conectividad y participación en proyectos de TD. Por otro lado, el indicador de tenencia TIC obtiene una puntuación promedio de 2,01; es decir, los centros educativos públicos en promedio 
alcanzan la mitad de la puntuación total de dicho indicador. Además, se presenta una similitud en los errores estándar de los promedios de los indicadores.

TABLA 4

Costa Rica: Estadísticos descriptivos de los indicadores y el índice, MEP, 2016

\begin{tabular}{lccccc}
\multicolumn{1}{c}{ Indicador e índice } & Min. & Máx. & M & SE de M & DE \\
\hline Indicador de Acceso & 0,00 & 6,00 & 3,27 & 0,019 & 1,19 \\
Indicador de Tenencia TIC & 0,00 & 4,00 & 2,01 & 0,016 & 0,97 \\
Índice de Acceso y Tenencia TIC & 0,00 & 9,75 & 5,29 & 0,031 & 1,91 \\
\hline
\end{tabular}

Fuente: elaboración propia.

Al desagregar el índice por algunas características generales, en la Tabla 5 se observa que el índice puntúa mayormente en centros educativos que se ubican en zona no vulnerable $(6,38)$, que se ubican en zona urbana $(5,96)$; además, se localizan en direcciones regionales centrales como San José Norte $(6,82)$ y en centros de secundaria técnica diurna $(7,24)$. Cabe destacar que se elaboran quintiles a partir del índice, en particular, en el primer quintil (menores índices) conformado por 744 centros, en promedio puntúan 2,39 y en el quinto quintil (mayor índice) constituido por 740 centros, estos obtienen una puntuación promedio de 7,73. En general, estos resultados muestran la existencia de brechas de acceso y tenencia de TIC en el sistema educativo público, específicamente en las direcciones regionales costeras $y$ fronterizas.

TABLA 5

Descriptivos del IAT TIC según características de los centros educativos públicos

\begin{tabular}{|c|c|c|c|}
\hline Variable & $\mathbf{N}$ & $\mathbf{M}$ & DE \\
\hline \multicolumn{4}{|c|}{ Centro en zona vulnerable* } \\
\hline No & 1141 & 6,38 & 1,49 \\
\hline Sí & 2592 & 4,81 & 1,88 \\
\hline \multicolumn{4}{|l|}{ Zona } \\
\hline Rural & 2301 & 4,86 & 1,86 \\
\hline Urbano & 1432 & 5,96 & 1,79 \\
\hline \multicolumn{4}{|l|}{ Dirección Regional } \\
\hline Aguirre & 96 & 4,78 & 1,85 \\
\hline Alajuela & 163 & 6,63 & 1,31 \\
\hline Cañas & 112 & 5,02 & 1,81 \\
\hline Cartago & 149 & 6,57 & 1,25 \\
\hline Coto & 278 & 4,19 & 2,08 \\
\hline Desamparados & 129 & 6,49 & 1,42 \\
\hline Grande de Térraba & 233 & 4,38 & 1,85 \\
\hline Guápiles & 176 & 4,93 & 1,86 \\
\hline Heredia & 102 & 6,81 & 1,22 \\
\hline Liberia & 102 & 5,18 & 1,97 \\
\hline Limón & 189 & 4,86 & 1,62 \\
\hline Los Santos & 65 & 5,99 & 1,44 \\
\hline Nicoya & 161 & 4,97 & 1,67 \\
\hline Occidente & 146 & 6,32 & 1,34 \\
\hline
\end{tabular}




\begin{tabular}{|c|c|c|c|}
\hline Variable & $\mathbf{N}$ & $\mathbf{M}$ & DE \\
\hline Peninsular & 65 & 5,02 & 2 \\
\hline Pérez Zeledón & 220 & 5,06 & 1,62 \\
\hline Puntarenas & 115 & 5,41 & 1,57 \\
\hline Puriscal & 98 & 5,52 & 1,59 \\
\hline San Carlos & 284 & 5,23 & 1,75 \\
\hline San José Central & 80 & 6,69 & 1,24 \\
\hline San José Norte & 73 & 6,82 & 1,16 \\
\hline San José Oeste & 59 & 6,71 & 1,20 \\
\hline Santa Cruz & 103 & 5,69 & 1,76 \\
\hline Sarapiquí & 123 & 5,28 & 1,67 \\
\hline Sulá & 77 & 2,50 & 1,88 \\
\hline Turrialba & 171 & 4,40 & 2,19 \\
\hline Zona Norte-Norte & 164 & 4,96 & 1,51 \\
\hline \multicolumn{4}{|l|}{ Tipo de centro } \\
\hline Preescolar & 870 & 5,80 & 1,57 \\
\hline Primaria & 3128 & 5,10 & 1,89 \\
\hline Secundaria académica diurna & 344 & 6,12 & 1,87 \\
\hline Secundaria académica nocturna & 49 & 5,91 & 1,40 \\
\hline Secundaria técnica diurna & 107 & 7,24 & 1,30 \\
\hline Secundaria técnica nocturna & 56 & 7,09 & 1,28 \\
\hline Secundaria Colegio Virtual & 10 & 6,12 & 1,94 \\
\hline CINDEA & 66 & 5,36 & 1,48 \\
\hline IPEC & 13 & 5,63 & 1,63 \\
\hline Otro tipo & 2 & 5,38 & 0,37 \\
\hline \multicolumn{4}{|l|}{ Quintil del índice } \\
\hline I & 744 & 2,39 & 0,96 \\
\hline II & 752 & 4,34 & 0,39 \\
\hline III & 748 & 5,50 & 0,29 \\
\hline IV & 749 & 6,47 & 0,29 \\
\hline V & 740 & 7,73 & 0,59 \\
\hline
\end{tabular}

La Figura 4 muestra el mapa coroplético de las Direcciones Regionales Educativas (DRE), se observa la puntuación promedio del IAT TIC por cada DRE, asimismo, se destaca que las menores puntuaciones pertenecen a las DRE de Sulá $(2,5)$, Coto $(4,19)$, Grande de Térraba $(4,38)$, Turrialba $(4,40)$ y Aguirre $(4,78)$. Las mayores puntuaciones provienen de las DRE de San José Norte $(6,82)$, San José Oeste $(6,71)$ y Heredia $(6,81) ;$ en su mayoría ubicadas en la región central del país. 
Figura 4. Mapa por DRE según puntuación promedio del IAT TIC MEP, 2016

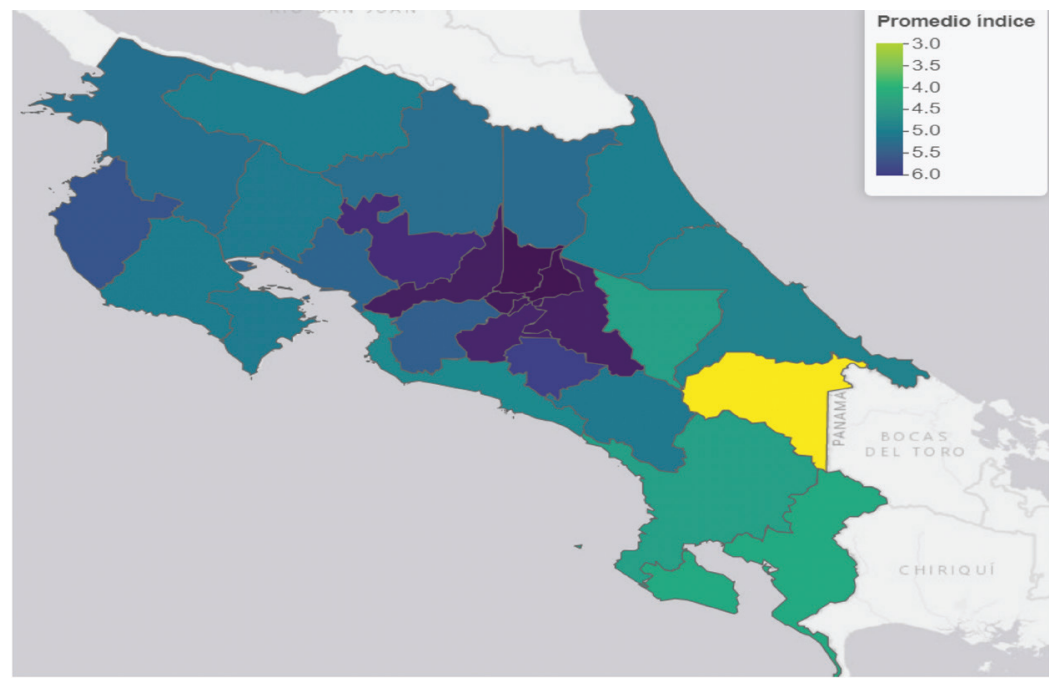

Fuente: elaboración propia.

Para profundizar el análisis del IAT TIC en las DRE, en la distribución porcentual de los centros por cada DRE, según los quintiles del índice, en la Figura 5 se muestra que la DRE de San José Oeste no muestra ningún centro en el I quintil. Se presentan DRE con porcentajes mayores al $40 \%$ de centros ubicados en el $V$ quintil, entre ellas: San José Norte (50,7\%), San José Oeste (40,7\%), Heredia (47,1\%), Cartago (40,3\%) y San José Central (48,8\%). Por otra parte, se evidencian DRE con más de $30 \%$ de los centros pertenecientes al I quintil, como Sulá (67,5\%), Coto (38,5\%), Turrialba (36,8\%), Grande de Térraba (33,9\%) y Aguirre (33,3\%).

Figura 5. Distribución de los centros educativos por DRA según quintil del IAT TIC

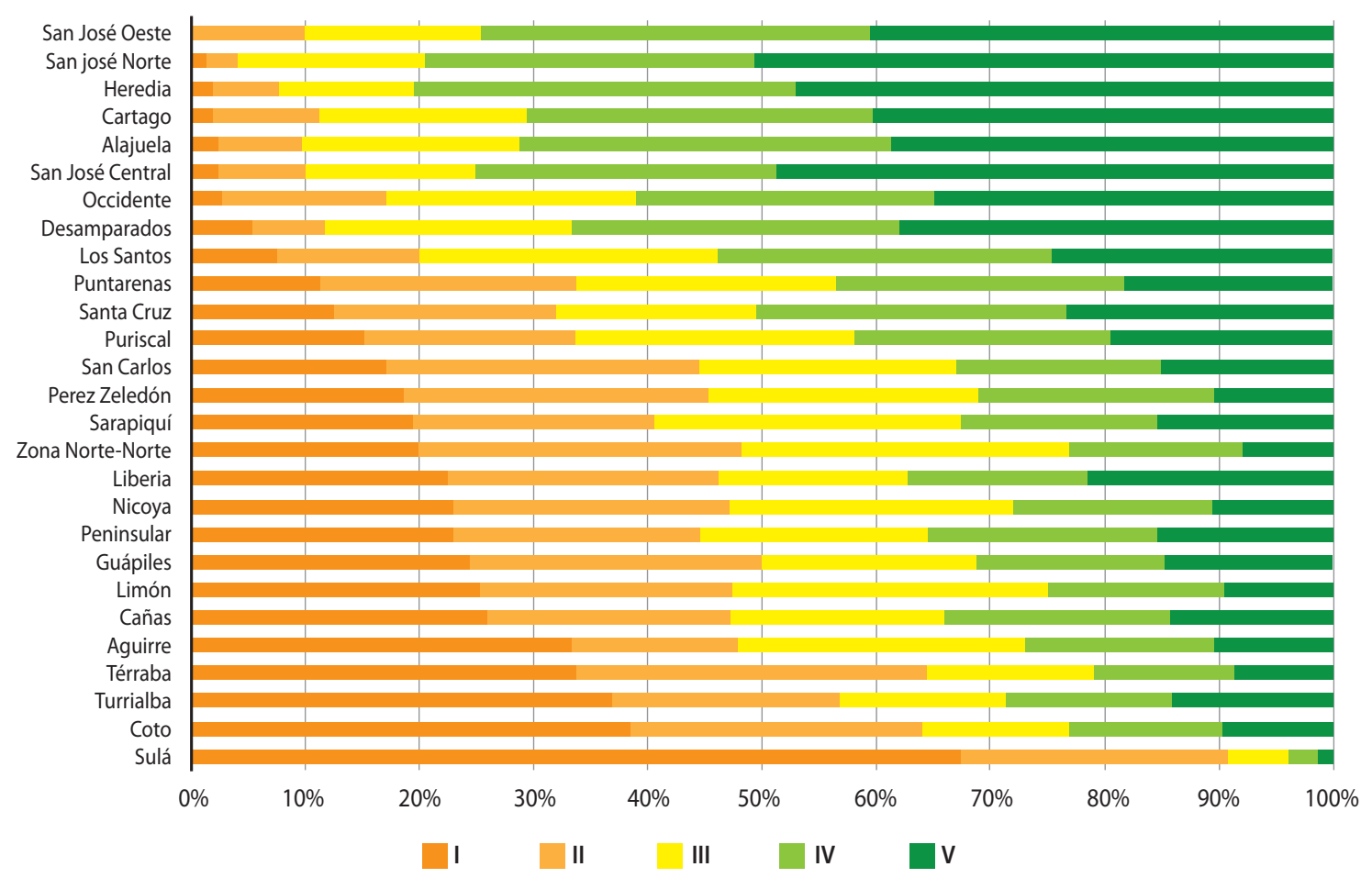

Fuente: elaboración propia. 
En el análisis de consistencia interna del IAT TIC, se estiman las correlaciones de Spearman entre el índice y los indicadores. En la Tabla 7, muestra que las correlaciones presentan magnitudes altas, entre el IAT TIC y el indicador de Acceso y Tenencia TIC se obtienen asociaciones de 0,877 y 0,871, respectivamente. Se presenta una moderada asociación entre los indicadores $(0,542)$, lo cual es pertinente.

TABLA 7

Correlaciones de Spearman de los indicadores y el índice, MEP, 2016

\begin{tabular}{lccc}
\multicolumn{1}{c}{ Indicador e índice } & Acceso & Tenencia TIC & Acceso y Tenencia TIC \\
a) Indicador de Acceso & 1 &, $42^{* *}$ &, $877^{* *}$ \\
b) Indicador de Tenencia TIC & & 1 &, $871^{* *}$ \\
c) Índice de Acceso y Tenencia TIC & & & 1 \\
\hline
\end{tabular}

**Correlación significante a un nivel de 0,01.

Fuente: elaboración propia.

Por otro lado, se realiza un análisis de factores de los dos indicadores construidos. Los resultados muestran que ambos miden una sola dimensión de acceso y tenencia TIC y, de manera conjunta explican aproximadamente el $78 \%$ de la varianza de esa dimensión. Asimismo, ambos indicadores presentan un alfa de Cronbach de 0,714 , lo cual indica una consistencia y confiabilidad interna aceptable para el IAT TIC. Los resultados del IAT TIC coinciden a nivel geográfico y DRE con la conectividad de los centros educativos de fibra óptica de los centros educativos en el 2016. En la Figura 6, se muestra que las regiones centrales del país se encuentran las velocidades más altas de conectividad.

Figura 6. Costa Rica: Mapa de Conectividad de Fibra Óptica en Centros Educativos Fuente: MEP (2016a)

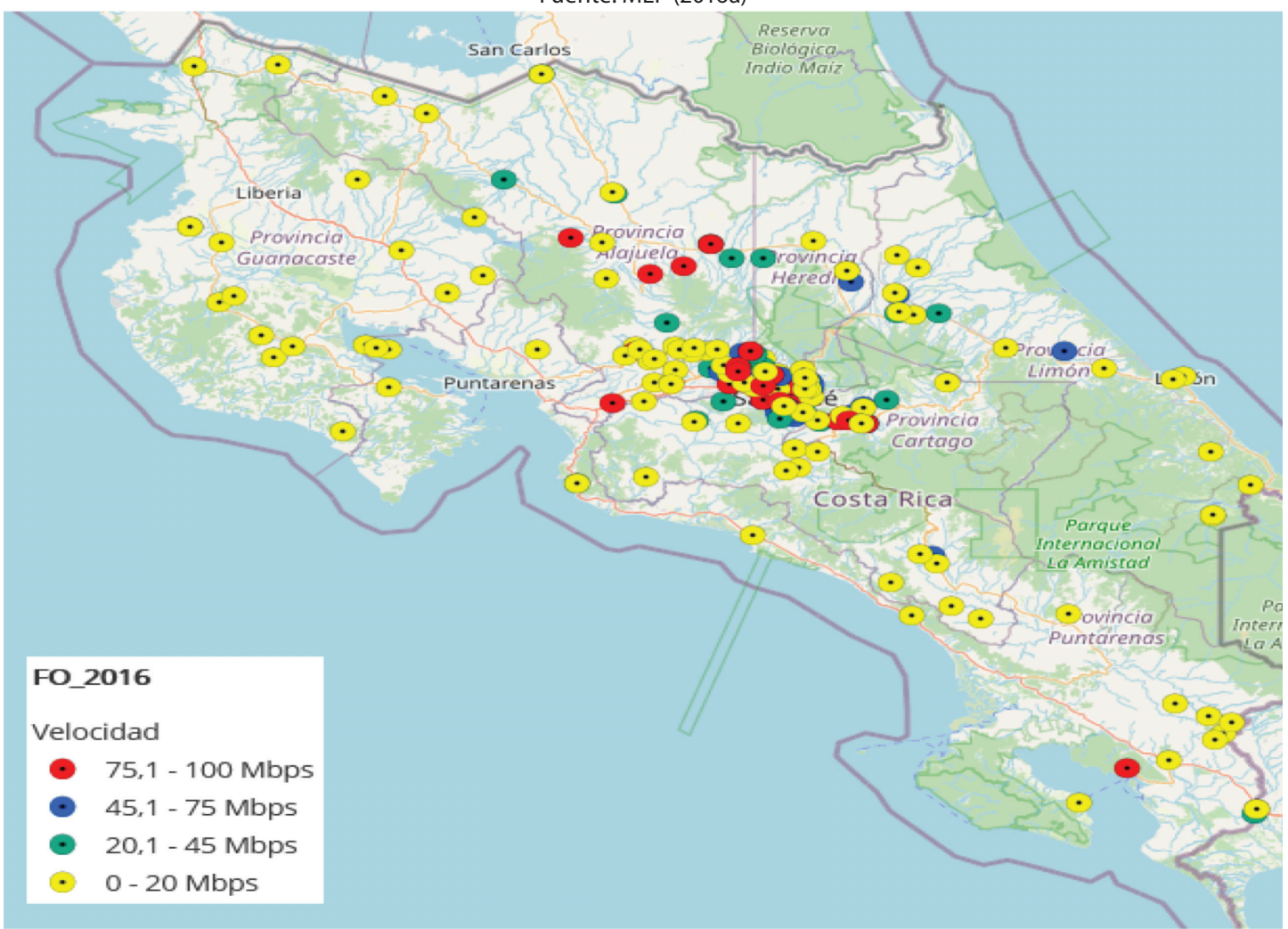


Además, el IAT TIC es consistente con los avances de conectividad en ese mismo año; pues en los centros educativos con un nivel de avance, la mayoría se trata de centros educativos de secundaria técnica. Y aún se presentaba la dificultad de conectividad en zonas de difícil acceso como Talamanca (Dirección Regional Sulá), también se mencionan esfuerzos por beneficiar a más centros educativos y cerrar la brecha digital (MEP, 2016b).

\section{SIINTESIS Y REFLEXIONES FINALES}

Esta propuesta de índice es una medida que visibiliza las condiciones de acceso y tenencia de dispositivos TIC de los centros educativos a nivel nacional, y se presenta como herramienta para quienes toman decisiones educativas. Esto como insumo para mejorar condiciones de infraestructura y conectividad, y así unan esfuerzos para disminuir la brecha digital.

El índice muestra bajas magnitudes promedio en direcciones regionales como Sulá, Coto, Térraba y Turrialba. Por otro lado, las mayores puntuaciones se presentan en direcciones regionales centrales como San José Norte, San José Oeste y Heredia. Lo que evidencia brechas entre las diferentes direcciones regionales y los retos por ampliar la conectividad en DRE fuera del área central. Además, se concluye que los centros educativos de secundaria técnica indican las mejores puntuaciones del índice, y en menor medida se hallan los de primaria, CINDEA e IPEC.

Es importante tomar en consideración que existen esfuerzos por efectuar mejoras en los accesos; por ejemplo, la Superintendencia de Telecomunicaciones (Sutel, 2020) reporta que el 93\% de los centros beneficiados por el Programa Comunidades Conectadas pertenecen al MEP, programa ejecutado con recursos del FONATEL para llevar conexión a Centros de Prestación de Servicios Públicos. Asociado a esto, en julio de 2021 se realiza una inspección para la primera escuela pública conectada en el marco del programa de FONATEL de la Red Educativa del Bicentenario (Sutel, 2021). Esta red es un proyecto en el cual participan el MEP y la Fundación Omar Dengo (FOD), que busca conectar en una única red de banda ancha a los centros educativos del país (FOD, s.f.). También se encuentra en discusión el Proyecto de Ley Programa Nacional de Alfabetización Digital, el cual busca utilizar recursos ociosos de FONATEL para disminuir la brecha (Muñoz, 2021).

Ante la ausencia de estudios de acceso y tenencia de TIC en el sistema costarricense a nivel nacional, se encuentra limitada la validez externa del índice. Se insta a las autoridades competentes a realizar y fomentar investigaciones con cobertura nacional y articular esfuerzos con otras instancias para abordar esta temática a profundidad; pues la limitante de los centros educativos en tener una edificación e instalaciones óptima y el acceso a internet son aspectos asociados a la brecha digital. Además, por la situación de la pandemia se propone calcular el índice con información reciente, ya que como se mencionó previamente, ha existido esfuerzos para disminuir la brecha digital; sin embargo, aún se presentan retos y desafíos en esta materia.

\section{REFERENCIAS}

Babbie, E. (2000). Fundamentos de la investigación social (2da. Ed.). México, DF: International Thompsom Editores.

Brenes, L. (2020). La BiblioCRA como espacio de innovación para la disminución de la brecha digital en el contexto de las comunidades rurales de Costa Rica. Revista conexiones: una experiencia más allá del aula, 12(2):43-48. 
Cabero Almenara, J., \& Ruiz-Palmero, J. (2017). Las Tecnologías de la Información y Comunicación para la inclusión: reformulando la brecha digital. Ijeri. International Journal of Educational Research and Innovation, 9:16-30. Recuperado de https://www.upo.es/revistas/index.php/IJERI/article/ view/2665/2222

Carreño, I. (febrero 6, 2020). Programas para reducir la brecha digital en Costa Rica superan metas en más de 100\%. DPL News. https://digitalpolicylaw.com/ programas-para-reducir-brecha-digital-en-costa-rica-superan-metas-en-mas-de-100/

Comisión Económica para América Latinay el Caribe. (2020). Universalizarelaccesoa las tecnologías digitales para enfrentar los efectos del COVID 19. Informe especial COVID 19 № 07. Recuperado de https://repositorio.cepal.org/bitstream/handle/11362/45938/S2000550_es.pdf?sequence=4\&isAllowed=y

Cuevas, F., Álvarez, V., y Ravasio, P. (2009). Brecha digital en la educación secundaria: El caso de los profesores costarricenses. Programa Sociedad de la Información y el Conocimiento. Recuperado de http:// repositorio.ucr.ac.cr/bitstream/handle/10669/629/Profesores.pdf?sequence=1\&isAllowed=y

Cuevas, F. y Núñez, N. (2016). Tecnologías digitales y educación. En Programa Sociedad de la Información y el Conocimiento (Ed.). Hacia la sociedad de la información y del conocimiento. PROSIC - Universidad de Costa Rica. Recuperado de http://www.prosic.ucr.ac.cr/sites/default/files/recursos/cap9_2016.pdf

Chanto, C., y Loaiga, J. (2020). Educandos universitarios: entre la brecha digital y el aprendizaje en tiempos de COVID-19. El caso de Universidad Nacional de Costa Rica (UNA), Sede Región Chorotega, Campus Liberia. Revista Nuevo Humanismo, 8(2):95-122. DOI: http://dx.doi.org/10.15359/rnh.8-2.5

Fernández, L. (2016). El uso didáctico y metodológico de las tabletas digitales en aulas de educación primaria y secundaria de Cataluña. Pixel-Bit. Revista de Medios y Educación, (48):9-25. DOI: http:// dx.doi.org/10.12795/pixelbit.2016.i48.01

Fundación Omar Dengo. (s.f.). Red educativa del bicentenario. Recuperado de https://fod.ac.cr/ red-educativa/

Gómez, D. A., Alvarado, R. A., Martínez, M., \& Díaz de León, C. (2018). La brecha digital: una revisión conceptual y aportaciones metodológicas para su estudio en México. Entreciencias: diálogos en la sociedad del conocimiento, 6(16):47-62.

González-Zabala, M. P, y Sánchez-Torres, J. (2012). Analysis of variables and indicators used to measure the information society. Ingeniare. Revista chilena de ingeniería, 20(3):433-446. DOI: https://dx.doi. org/10.4067/S0718-33052012000300017

Hawash, R., \& Lang, G. (2020). Does the digital gap matter? Estimating the impact of ICT on productivity in developing countries. Eurasian Economic Review, 10(2):189-209. DOI: https://doi.org/10.1007/ s40822-019-00133-1

López-Mondéjar, L. M. (2018). El uso de la televisión en el hogar y en el aula de Educación Primaria. Educatio Siglo XXI, 36(1 Mar-Jun):195-214. DOI: http://dx.doi.org/10.6018/j/32423

Lugo, M. (Coord.). (2016). Entornos digitales y políticas educativas. Dilemas y certezas. UNESCO. https://www.buenosaires.iiep.unesco.org/es/publicaciones/ entornos-digitalesy-politicas-educativas-dilemas-y-certezas

Lugo, M. Kelly, V. (2010). Tecnología en educación: ¿Políticas para la innovación? Instituto de Planeamiento de la Educación IIPE-UNESCO. Recuperado de https://oei.org.ar/ibertic/evaluacion/sites/default/ files/biblioteca/13_tecnologia_en_educacion_lugo_kelly.pdf

Lugo, M. Kelly, V. (2011). La matriz TIC: una herramienta para planificar las tecnologías de la información en las instituciones educativas. Instituto Internacional de Planeamiento de la Educación IIPE-UNESCO. Recuperado de https://unesdoc.unesco.org/ark:/48223/pf0000371335

Ministerio de Ciencia, Tecnología y Telecomunicaciones. (2019). Índice de brecha digital 2016-2016. MICITT. Recuperado de https://www.micit.go.cr/sites/default/files/indice_de_brecha_digital_2016-2018_0.pdf 
Ministerio de Educación Pública de Costa Rica (2016a). Conectividad de Fibra Óptica en Centros Educativos. Recuperado de https://sigmep.maps.arcgis.com/apps/StorytellingSwipe/index.html ?appid=126b81407a184bc68646cfdbdcb07bfd\#

Ministerio de Educación Pública de Costa Rica. (2016b). MEP presenta avances en conectividad desde 18 puntos de Costa Rica. Recuperado de https://www.mep.go.cr/noticias/ mep-presenta-avances-conectividad-desde-18-puntos-costa-rica

Ministerio de Educación Pública de Costa Rica. (s.f.). Direcciones Regionales de Educación. Recuperado de https://www.mep.go.cr/organigrama/direcciones-regionales-de-educacion

Montero, E. (2008). Escalas o Índices para la medición de constructos: El dilema del analista de datos. Avances en medición, 6(1):17-26.

Morales, M. A. (2020). Las brechas digitales en México: Un balance pertinente *. El Trimestre Económico, 87(2):367-402. doi:http://dx.doi.org.ezproxy.sibdi.ucr.ac.cr:2048/10.20430/ete.v87i346.974

Moreira, J., Palomares, J., Serrano, R., \& López, J. (2017). Un breve análisis de la brecha digital de acceso en el Ecuador. Jornadas SARTECO. Recuperado de https://zenodo.org/record/1025815

Muñoz, D. (21 de mayo de 2021). Plan de ley de Alfabetización Digital se traslada a Comisión de Hacendarios. Semanario Universidad.

Núñez, N. (2018). Tenencia, uso y actitudes hacia las TIC. Estudio a los directores de primaria y secundaria de centros educativos públicos. En Programa Sociedad de la Información y el Conocimiento (Ed.). Hacia la sociedad de la información y del conocimiento. PROSIC - UCR. Recuperado de http:// www.prosic.ucr.ac.cr/informe-hacia-la-sociedad-de-la-informacion-y-elconocimiento-2018

Organización de las Naciones Unidas para la Educación, la Ciencia y la Cultura. (2009). Medición de las tecnologías de la información y la comunicación (TIC) en educación. UNESCO. Recuperado de https://www.cepal.org/socinfo/noticias/documentosdetrabajo/0/40930/188309s.pdf

Ramírez, D. (agosto 8, 2021). La brecha digital en Costa Rica: luchando contra las adversidades del entorno virtual. Delfino. Recuperado de https://delfino.cr/2021/08/ la-brecha-digital-en-costa-rica-luchando-contra-las-adversidades-del-entorno-virtual

Severin, E. (2011). Tecnologías para la Educación (TEd): un marco para la acción. Banco Interamericano de Desarrollo. Recuperado de https://publications.iadb.org/es/publicacion/15177/ tecnologias-para-la-educacion-ted-un-marco-para-la-accion

Solórzano, K. (2008). Indicadores TIC en Costa Rica. Recuperado de https://www.itu.int/ITU-D/ict/ events/dominicanrep08/material/CostaRica.pdf

Superintendencia de Telecomunicaciones. (2020). Estadísticas del Sector telecomunicaciones. Recuperado de https://www.sutel.go.cr/sites/default/files/sutel_informe_estadistico_2020_digital.pdf

Superintendencia de Telecomunicaciones. (2021). Guanacaste celebra la primera escuela conectada a la Red Educativa del Bicentenario a través de FONATEL. Recuperado https://www.sutel.go.cr/noticias/ comunicados-de-prensa/guanacaste-celebra-la-primera-escuela-conectada-la-red-educativa-del

Tiana, A. (2021). Los sistemas de indicadores: una radiografía de la educación. En M. Kisilevsky y E. Roca (Eds.). Indicadores, metas y políticas educativas. Madrid: Organización de Estados Iberoamericanos para la Educación, la Ciencia y la Cultura. Recuperado de https://www.oei.es/uploads/files/microsites/28/140/eval2.pdf

Vázquez, P. N., \& Ramírez, M. M. O. (2016). Una Aproximación a un Sistema de Indicadores para Evaluar la Integración de las TIC en las Instituciones de Educación Superior. Areté: Revista Digital del Doctorado en Educación de la Universidad Central de Venezuela, 2(4):63-89. Recuperado de http:// saber.ucv.ve/ojs/index.php/rev_arete/article/view/11878/11570 